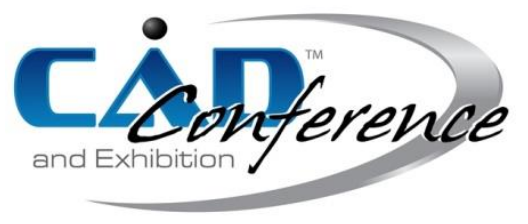

Title:

Real-Time NC Simulation with Instantaneous and Localized Cutting Force Prediction

Authors:

Randy Chang, callmerandy0979@gmail.com, National Chung Cheng University

Si-Yuan Wang, d0348089@itd.fcu.edu.tw, National Chung Cheng University

Hong-Tzong Yau, imehtyccu@gmail.com, National Chung Cheng University

Keywords:

NC Simulation, Cutting Force, GPU, Tool Life, Digital Twin, CPS.

DOI: 10.14733/cadconfP.2021.308-313

Introduction:

In the wave of Industry 4.0, smart manufacturing must integrate tools such as the Internet of Things (IoT), cloud computing, and big data collection and analysis, but more importantly, it must develop and use a virtual physical system (Cyber Physical System, CPS) to simulate the entire manufacturing processes. Machine tool is the mother of manufacturing. CPS in Industry 4.0 must first be able to simulate the complete real situation of machine tool processing, not only to simulate the processing path, but also to estimate cutting force, cutting error, even temperature rise and dynamic error, etc. The physical quantity becomes a true digital twin. The current commercial cutting simulation software can only simulate the machining geometry of the NC path, and cannot really achieve the goal of CPS requirements. Therefore, the purpose of this research is to develop a next-generation machining simulation system and use GPU parallel computing to realize a real-time virtual machining system to meet the constraints of machining accuracy and stable cutting force and to plan the optimized machining tool path and process parameters. Regarding the optimized tool path, the path interval can be recalculated by setting the surface accuracy of the workpiece to obtain the most efficient path under the condition of accuracy. For the optimized machining feed rate, through real-time multi-axis machining simulation and GPU parallel computing, the instantaneous cutting force load condition in the path is calculated in real time, and the machining feed rate is adjusted for the purpose of maintaining a stable cutting force. The real-time simulation method can not only calculate the best cutting conditions to increase cutting efficiency, but also avoid the increase in machining costs caused by unexpected errors such as collisions, and maintain the stability of the machine during machining and extend the tool and Machine life.

\title{
Related work:
}

Altintas [1] proposed a cutting force model for ball-end milling in 1998. By considering the instantaneous regenerative chip load, the structure transfer function and the geometry of the ball-end milling process were mathematically modeled. In addition to Altintas, Wang [2] and Kao [3] also proposed a more complete derivation of the cutting force coefficients based on the same cutting force model as Altintas, and verified the accuracy of the cutting coefficients by cutting experiments and measurements. However, similar to the papers by Altintas, almost all researches were conducted under the full immersion of the cutting tool and the experiments were also conducted in the way of slot milling. However, in the actual machining of aerospace workpieces or molds, due to the geometric changes of the workpiece surface, the contact area between the tool and the workpiece is constantly changing. The geometry of the engagement angle and the actual engagement area is very complicated, resulting in complex and varying cutting forces. So to truly estimate the instantaneous cutting force 
during multi-axis machining, the cutting force analysis must be combined with real-time multi-axis NC simulation in order to truly achieve the required goal of CPS.

Commercial software such as VeriCut uses material removal rate (MRR) to calculate cutting force. This is because MRR is easier to calculate during cutting simulation and can achieve real-time results. However, the MRR model cannot accurately estimate the instantaneous cutting force, especially the maximum instantaneous cutting force is usually underestimated. In addition, the MRR model cannot estimate the direction of the cutting force and the components of each axis. Therefore, Erdim [4] compared the results of the MRR model and the mechanistic model to estimate the cutting force, and pointed out that the correct cutting force analysis should still be carried out in the way of the mechanistic cutting force model to adjust the feed rate, in order to achieve the goal of improving processing efficiency and protecting tools and machines.

\section{Main Idea:}

It can be known from the above-mentioned literature that the geometric modeling technology in the virtual environment is very important for the accuracy of machining simulation. And CPS (Cyber Physical System) was proposed to collect and manage data measured by sensors on the machine, and use big data analysis and machine-Internet functions to achieve smart manufacturing. In the past, the simulation processing system used to simulate the NC tool path, and could not consider the various variables of the machine, including cutting force, path adjustment, etc., which may cause the risk of tool collision and overcutting during real machining. Therefore, in this work we develop a real-time NC simulation system which simulates the instantaneous cutting conditions during machining, and calculates the instantaneous cutting force for each different axis, so as to achieve the purpose of accurately grasping the machine status, thereby improving the machining efficiency while protecting the safety of the machine tool.

The purpose of this research is to understand the machine and actual processing conditions through machining simulation, optimize processing parameters and paths, and develop a cutting simulation system for virtual machining. The system will use solid modeling to calculate the geometric changes between the tool and the workpiece, estimate the instantaneous cutting force, and evaluate the machinability from the estimated instantaneous cutting force value and adjust it to suitable cutting conditions. The cutting adjustment for the machine condition includes the following three aspects:

1. Through real-time cutting force information, simulate the impact of various cutting conditions on processing, assist users in adjusting processing parameters and improve processing efficiency.

2. Pre-calculate the corner and curve cutting feed rate value and resulting cutting force in the processing path, and implement acceleration and deceleration planning on the path according to the preview results to improve the reliability of the simulation system.

3. Using the proposed system, according to the information provided by the NC code and the CAD geometry, instantaneous cutting force calculation and automatic feed rate adjustment are carried out to avoid damage to the workpiece and the tool.

\section{NC Simulation}

When calculating the cutting force in milling, in order to make the simulation results more accurate, it is necessary to calculate the contact surface between the tool and the workpiece in real time, because the cutting geometry is complex and the contact area with the tool changes greatly during machining. As in our previous work [5], implicit functions are used to represent the geometry of the tool. The ball milling cutter can use the union of the cylinder and the sphere to express the related implicit function, as shown in Fig. 1. Assuming that the tool is parallel to the Z-axis direction and is translated to the center of the sphere according to the center point of the coordinate system, the implicit function of the ball milling cutter can be expressed as Eq. (1):

$$
\mathrm{F}(\mathrm{X}, \mathrm{Y}, \mathrm{Z})=\left\{\begin{array}{c}
\max \left\{\operatorname{abs}(Z)-L, \quad X^{2}+Y^{2}-R^{2}\right\} \text { of } Z \geq 0 \\
X^{2}+Y^{2}+Z^{2}-R^{2}, \quad \text { otherwise }
\end{array}\right.
$$

where $R$ is the radius of the tool, and $L$ is the distance along the tool axis from the center point, as shown in Fig. 1 During the cutting process, it is necessary to continuously reconstruct the STL surface 
model, so that the new surface after cutting can be visualized. To build a surface triangle mesh from the voxel model, the classic Marching Cube algorithm is used.

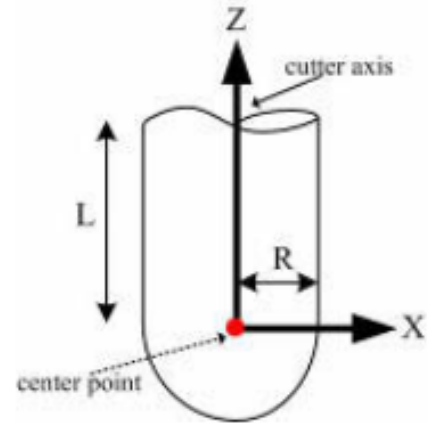

Fig. 1: Cutter coordinate system.

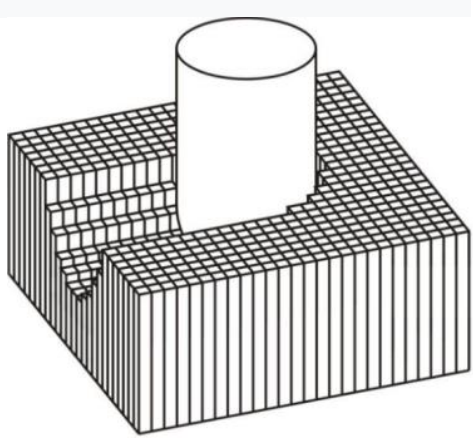

Fig. 2: Dexelized NC simulation.

\section{Cutting force evaluation}

The cutting force is estimated by projecting the cutting force components in the tool coordinate system onto the corresponding machine coordinate system axis to obtain the actual force load generated on the machine. Based on system dynamics, the cutting force in each path point needs to be determined in advance so that the cutting parameters can be optimized based on the results. The NC code of the machining path generated by the CAM system can segment the complex tool path. Through the cutting force estimation of the data content of each CL point, the cutting force can be maintained at a safe amount, thereby optimizing other cutting conditions and improving efficiency.

The milling process is expressed using cylindrical milling cutter coordinates and the workpiece coordinate system, and the cutting edge angle is calculated according to the definition of milling cutter coordinates. $\beta$ is the helix angle of the ball-end mill. $\phi$ is the rotation angle of the tool at different heights at $\mathrm{z}$ as shown in Eq. (2):

$$
\phi=\frac{z \times \tan (\beta)}{R}
$$

This relationship can be used to determine whether the cutting edge at depth $\mathrm{z}$ is within the effective cutting range, $j$ is the number of edges and $N$ is the number of edges, where $\phi_{j}$ is as follows:

$$
\phi_{j}=\theta_{s t}+(N-1) \frac{2 \pi}{N}-\frac{z}{R_{0}} \tan \beta
$$

Tangential force $\left(\mathrm{d} F_{t j}\right)$, axial force $\left(\mathrm{d} F_{r j}\right)$, radial force $\left(\mathrm{d} F_{a j}\right)$, the cutting force per unit $(\mathrm{j})$ can be written as Eq. (4)

$$
\begin{gathered}
\mathrm{d} F_{t j}=K_{t e} * d S+K_{t c} * h(\phi, \kappa) * d b \\
\mathrm{~d} F_{r j}=K_{r e} * d S+K_{r c} * h(\phi, \kappa) * d b \\
\mathrm{~d} F_{a j}=K_{a e} * d S+K_{a c} * h(\phi, \kappa) * d b
\end{gathered}
$$

Among them, the parameters $K_{t c}, K_{r c}, K_{a c}$ are the cutting force coefficients generated by the shearing force and the cutting force coefficients $K_{t e}, K_{r e}, K_{a e}$ generated by the tool edge surface rubbing the workpiece in the tangential, radial and axial directions, respectively. The projection length $(d b)$ in the infinitely small cutting groove along the cutting speed direction is calculated as $d b=d z \times \sin (\kappa)$, and the cutting depth $(\mathrm{dz})$ of the tool for each axial element $j$ is calculated. According to the change of the rotation angle $\phi_{j}$, the cylindrical coordinates of the vector from the point center of the cutting edge to the cutting point can be defined as Eq. (5): 


$$
\begin{gathered}
\vec{r}_{j}=x_{j} \vec{\imath}+y_{j} \vec{\jmath}+z_{j} \vec{k} \\
=r\left(\phi_{j}\right)\left(\sin \phi_{j} \vec{\imath}+\cos \phi_{j} \vec{\jmath}\right)+z\left(\phi_{j}\right) \vec{k}
\end{gathered}
$$

The length of the spiral cutting edge of the tool can be deduced from the geometric position (6):

$$
d S=|d r|=\sqrt{r^{2}(\phi)+\left(r^{\prime}(\phi)\right)^{2}+\left(z^{\prime}(\phi)\right)^{2}} d \phi
$$

From the geometric position information of the tool, the chip thickness $h$ of the tool during cutting is calculated as shown in the following Eq. (8), where $S_{t j}$ is the feed per edge of the tool, $\kappa$ is the immersion angle which is deduced according to the change of geometric position, which can be expressed as Eq. (7)

$$
\begin{gathered}
\kappa=\sin ^{-1}\left(\sqrt{R_{0}^{2}-\left(R_{0}-z\right)^{2}} / R_{0}\right) \\
h\left(\phi_{j}\right)=s_{t j} \sin \phi_{j} \sin \kappa
\end{gathered}
$$

Using geometric transformation, we can convert the component forces into the Cartesian coordinate system, and obtain the forces representing the three directions of $F_{x}, F_{y}, F_{z}$.

$$
\left[\begin{array}{l}
d F_{x} \\
d F_{y} \\
d F_{z}
\end{array}\right]=\left[\begin{array}{ccc}
-\sin \phi \sin \kappa & -\cos \phi & -\sin \phi \cos \kappa \\
-\cos \phi \sin \kappa & \sin \phi & -\cos \phi \cos \kappa \\
-\cos \kappa & 0 & -\sin \kappa
\end{array}\right]\left[\begin{array}{l}
d F_{r} \\
d F_{t} \\
d F_{a}
\end{array}\right]
$$

Finally, the cutting force per unit in the cutting range is added, and the total cutting force in each axial direction is obtained.

\section{Instantaneous cutting force simulation}

What we need is to calculate the correct instantaneous cutting force in real time in the machining simulation. Although the estimated cutting force based on the mechanistic machining model is accurate, the amount of calculation is huge, and it must be integrated with the multi-axis cutting simulation to correctly calculate the engaged area of the machined surface. Therefore, it must also be combined with the GPU parallel computation for real-time simulation to meet CPS requirements. In this work, we use OpenGl compute shader to achieve GPU parallel computing running on a PC with Intel i5-3570 3.4 Ghz CPU and an Nvidia GeForce GTX 1650. Throughout the simulation, FPS is maintained around 30, sufficient for real-time simulation and display.

According to the NC simulation, the engaged area where the tool really contacts the workpiece during cutting can be obtained, and the instantaneous cutting force in each axial direction at the contact point of different depth of cut $\mathrm{z}$ and different rotation angle $\phi$ in each engaged area can be calculated.

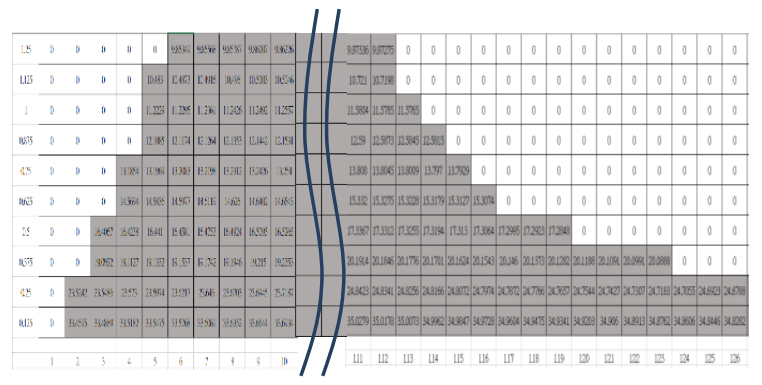

Fig. 3: Distribution of instantaneous cutting force.

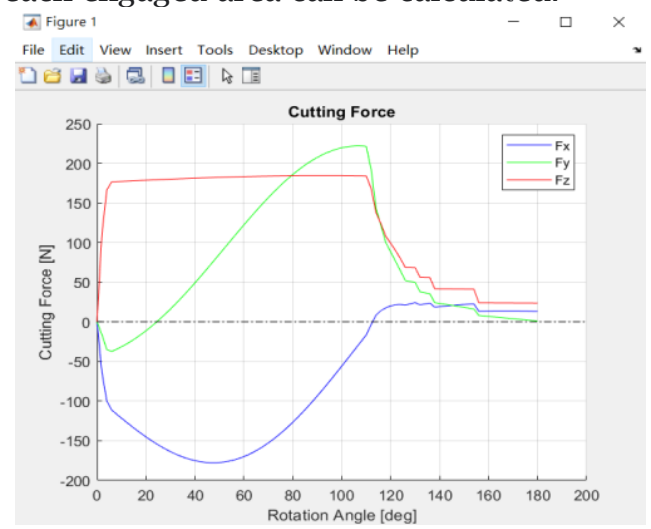

Fig. 4: Three components of I-C force. 
Fig. 3 shows that the engaged area is first obtained (dark area) and the localized and instantaneous cutting force is calculated at different rotation angles and $\mathrm{z}$ positions. Fig.4 shows the resulting three component forces at different rotation angles. Combined with GPU for parallel computing, the cutting force information of all contact points in each engaged area can be calculated in real time, and the cutting conditions during machining can be monitored and modified in real time. Fig. 5 shows the realtime NC simulation with instantaneous cutting force calculation in action. The left shows the visualization of the machined surface. The right shows the instantaneous localized cutting force at different rotation angles and $\mathrm{z}$ heights.

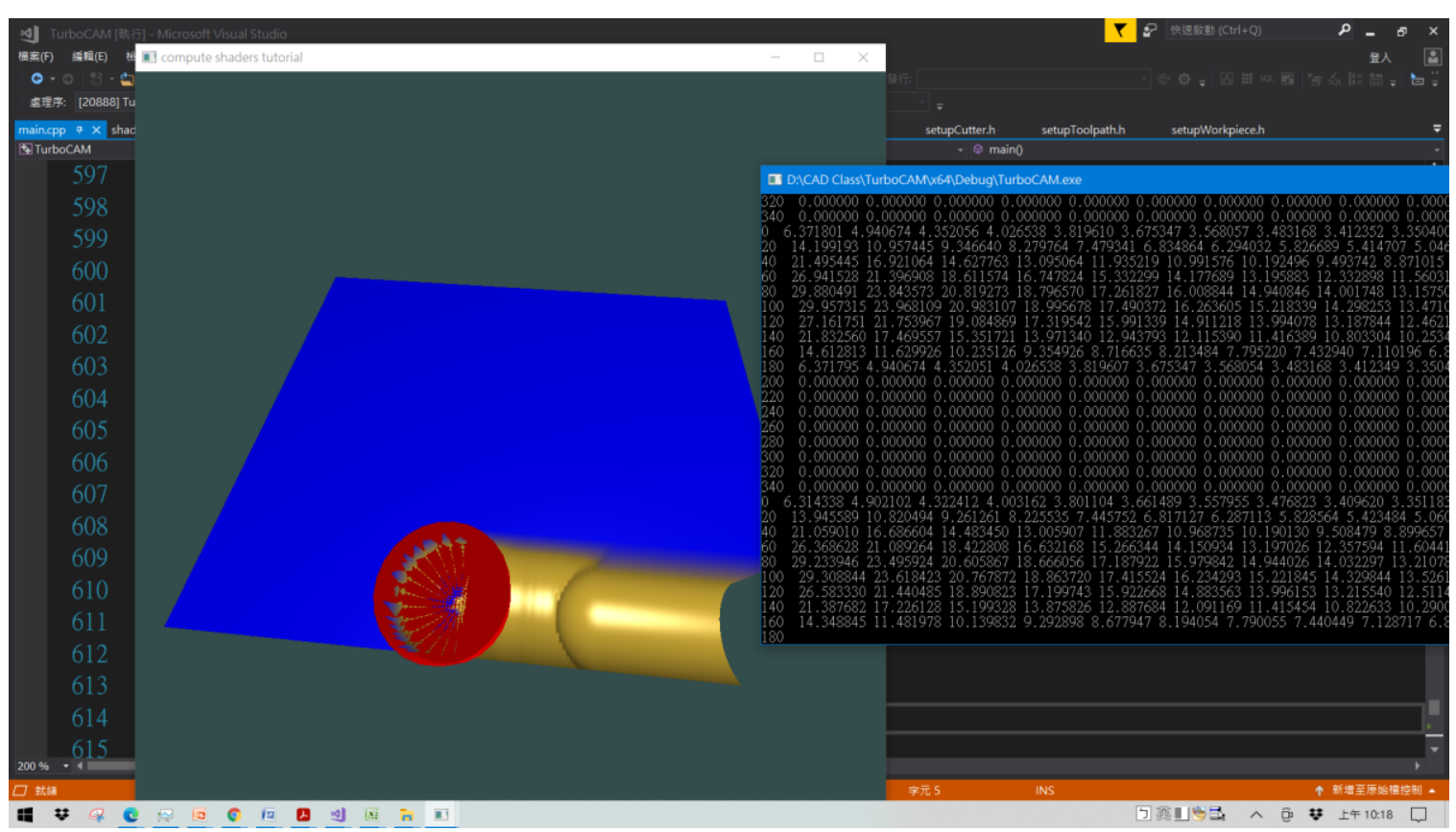

Fig. 5: Real-time NC simulation with instantaneous cutting force calculation in action.

Conclusions:

Combining intelligent manufacturing CPS and parallel computing, optimizing processing parameters and tool paths through the constraints of processing conditions and machines, can instantly generate new processing paths and cutting conditions, cutting in the shortest time and lowest processing cost, and It can also maintain the surface accuracy of the workpiece, so as to achieve the simulation of the integration of virtual and real. This work takes the virtual machining system as the main focus, optimizes the parameters and paths in the machining process, and adds the concept of Industry 4.0 to machine-specific simulation of machining simulation. Through more precise simulation, different customized simulation results can be achieved with different machines, and the machine status can be more accurately estimated, thereby enhancing smart manufacturing capabilities and the added value of machine tools.

\section{References:}

[1] Altıntas, Y.; Lee, P.: Mechanics and Dynamics of Ball End Milling, Journal of Manufacturing Science and Engineering, 120(4), 1998, 684-692. https://doi.org/10.1115/1.2830207

[2] Wang, J.J.; Zheng, C.M.; Identification of shearing and ploughing cutting constants from average forces in ball-end milling., International Journal of Machine Tools and Manufacture, 2002, 695-705. https://doi.org/10.1016/S0890-6955(02)00002-0 
[3] Kao, Y.-C.; Chen, M.-S.; Nguyen, N.-T.: A combination method of the theory and experiment in determination of cutting force coefficients in ball-end mill processes, Journal of Computational Design and Engineering, 2(4), 2015, 233-247. https://doi.org/10.1016/j.jcde.2015.06.005

[4] Erdim, H.; Lazoglu, I.; Ozturk, B.: Feedrate scheduling strategies for free-form surfaces, International Journal of Machine Tools and Manufacture, 46(7), 2006, 747-757. https://doi.org/10.1016/j.ijmachtools.2005.07.036

[5] Yau, H.T.; Tsou, L.S.: Efficient NC Simulation for Multi-Axis Solid Machining with a Universal APT Cutter, ASME Transaction, Journal of Computing and Information science in Engineering, 9(2), Article Number: 021001, Jun 2009. https://doi.org/10.1115/1.3130231 